\title{
Backcalculation of Non-Linear Pavement Moduli Using Finite-Element Based Neuro-Genetic Hybrid Optimization
}

\author{
Kasthurirangan Gopalakrishnan*
}

Department of Civil, Construction and Environmental Engineering, Iowa State University, Ames, Iowa, USA

\begin{abstract}
The determination of pavement layer stiffness is an essential step in evaluating the performance of existing road pavements and in conducting pavement design and analysis using mechanistic approaches. Over the years, several methodologies involving static, dynamic, and adaptive processes have been developed and proposed for obtaining in-situ pavement layer moduli from Falling Weight Deflectometer (FWD) test deflection data through inverse analysis and parameter identification routines. In this paper, a novel pavement analysis toolbox combining the strengths of Finite Element (FE) modeling, Neural Networks (NNs), and Genetic Algorithms (GAs) is described. The developed user-friendly automated pavement evaluation toolbox, referred to as Neuro-Genetic Optimization Toolbox (NGOT) can be used on a real-time basis for accurate and rapid transportation infrastructure evaluation. It is shown that the developed toolbox backcalculates non-linear pavement layer moduli from actual field data with better accuracy compared to regression and conventional backcalculation approaches.
\end{abstract}

\section{INTRODUCTION}

According to recently published data [1], billions of U.S. dollars are needed annually to improve transportation infrastructure conditions nationally. Thus, consistent, costeffective, and accurate monitoring of pavement is necessary for improving the performance and serviceability of pavements and to schedule proactive pavement repair and maintenance activities.

About $93 \%$ of the paved roads in the US are reported to be composed of flexible pavement [2]. Flexible pavements are multi-layered, heterogeneous structures that are designed to "flex" under repeated traffic loading. A typical flexible pavement structure consists of the surface course (typically Hot-Mix Asphalt) at the top, underlying base and subbase (optional) courses (typically unbound granular material), and a subgrade (typically soil) at the bottom.

In the field, Non-Destructive Testing (NDT) of in-service pavements using a Falling Weight Deflectometer (FWD) equipment is carried out to measure the deflection response of the pavement structure to applied dynamic load that simulates a moving wheel. The deflected shape of the basin is predominantly a function of the thickness of the pavement layers, the moduli of individual layers, and the magnitude of the load. The surface deflections are typically measured at radial offsets of $0 \mathrm{~mm}$ (D0), $300 \mathrm{~mm}$ (D1 or D300), $600 \mathrm{~mm}$ (D2 or D600), $900 \mathrm{~mm}$ (D3 or D900), $1200 \mathrm{~mm}$ (D4 or D1200), and $1500 \mathrm{~mm}$ (D5 or D1500) from the center of FWD load plate.

"Backcalculation" is the accepted term used to identify a process whereby the elastic (Young's) moduli of individual

*Address correspondence to this author at the Civil Engineering, 354 Town Engineering Bldg. Iowa State University, Ames, IA 50011-3232; USA; Tel: (515) 294-3044; Fax: (515) 294-8216; E-mail: rangan@iastate.edu pavement layers are estimated based upon measured FWD surface deflections. As there are no closed-form solutions to accomplish this task, a mathematical model of the pavement system (called a forward model) is constructed and used to compute theoretical surface deflections with assumed initial layer moduli values at the appropriate FWD loads (referred to as forward calculation). Through a series of iterations, the layer moduli are changed, and the calculated deflections are then compared to the measured deflections until a match is obtained within tolerance limits. Most of the commercial backcalculation programs currently in use utilize an Elastic Layer Program (ELP) as the forward model to compute the surface deflections. Many studies have addressed the interpretation of FWD pavement deflection measurements as a tool to characterize pavement-subgrade systems [3-5].

The backcalculated in-situ pavement layer moduli from measured deflections (inverse analysis) are by themselves indicators of pavement layer condition as well as necessary inputs for conducting mechanistic pavement structural analysis and remaining life calculations [6, 7]. Over the years, several techniques have been proposed for back-calculation of pavement layer moduli such as the least-squares (parameter identification), database search, Neural Networks (NNs), neuro-fuzzy systems, and recently Genetic Algorithms (GAs) [8-21].

The hybrid approach presented in this paper represents the latest development in backcalculating the mechanical properties of flexible pavement systems. This innovative approach takes advantage of the combined efficiency and accuracy achieved by integrating advanced pavement numerical modeling schemes, computational intelligence based surrogate mapping techniques, and heuristics based global optimization strategies, and yet provides a userfriendly pavement evaluation toolbox for the pavement engineer to use on a real-time basis for accurate infrastructure evaluation. 


\section{PAVEMENT LAYER MODULI BACKCALCULA- TION}

Considering the complex nature of the backcalculation problem, numerous approaches have been proposed and efforts have also been made to develop a standardized approach [22, 23]. The discrepancies among the numerous backcalculation techniques developed for the backcalculation of pavement layer moduli arise from the type of the pavement (forward) response model and the optimization procedure utilized for the determination of appropriate layer modulus values [21]. Existing methods of backcalculation rely on some assumptions and simplifications that should be made to facilitate the backcalculation process. Some of the major difficulties faced by researchers in using the different commercial backcalculation programs include: (1) ability to handle only limited and idealistic solutions due to closedform nature of solutions, (2) longer computational time in using conventional optimization techniques for backcalculation, (3) convergence of backcalculation solutions to local optima or non-unique nature of solutions depending on seed moduli (initial values), etc. [22].

Most of the conventional commercial backcalculation programs involve Multi-Layer Elastic Theory (MLET) in their forward calculation routines and assume that pavement materials are linear-elastic, homogenous, and isotropic resulting in unrealistic backcalculated pavement layer moduli. Several research studies have shown that pavement geomaterials used in the underlying pavement layers exhibit non-linear, stress-sensitive behavior under repeated traffic loading. Unbound aggregates used in pavement base/subbase course exhibit stress-hardening and fine-grained soils show stress-softening-type behavior [24-26]. Research studies have shown that non-linear analysis using FE based approach increases the precision of the forward model [21]. Majority of the commercial backcalculation programs employ an iterative approach (see Fig. 3) which is known to suffer from limitations such as dependency on the initial seed moduli and the possibility of local minimum solutions [27]. Other limitations include computational ineffectiveness or lack of robustness leading to divergence in some cases where no solution is obtained, requiring a high level of userinvolvement during the backcalculation process making it less amenable to automation, number and thickness of layers used in the analyzed pavement system, etc. [17].

To overcome the limitations associated with existing commercial backcalculation programs, a new toolkit referred to as Neuro-Genetic Optimization Toolbox (NGOT) was developed through FE-based neuro-genetic integrated systems approach. Such a hybrid approach towards backcalculation has many advantages which include realistic prediction of non-linear pavement layer moduli, rapid prediction ability and the provision to model uncertainties in the deflection data including noise, errors, etc. and derivation of global optimum solutions. In addition, such an intelligent, yet user-friendly toolbox can provide pavement engineers and designers with the ability to rapidly evaluate the infrastructure condition in real-time without requiring them to have in-depth knowledge of the actual modeling of the problem.

\section{NEURO-GENETIC OPTIMIZATION}

The framework of NGOT as discussed in this paper is shown in Fig. (2). As mentioned previously, depending on the problem under consideration, the inputs and outputs will vary. The problem under consideration is to backcalculate pavement layer moduli from non-destructive test deflection data acquired using the FWD device. The major modules integrated into NGOT are the GA module, NN module and FE module. Each of these modules with a brief introduction and background and the steps involved in developing the NGOT toolbox are discussed in the following sub-sections.

\section{Genetic Algorithms}

Genetic algorithms are a part of evolutionary computing, a rapidly growing area of artificial intelligence. Categorized as global search heuristics, GAs use techniques inspired by evolutionary biology such as inheritance, mutation, selection, and crossover (also called recombination). Being robust search and optimization techniques, GAs are finding applications in a number of practical problems where calculus-based search methods are inefficient in searching for the optimal solution in a complex multi-modal search space [28-31]. In recent years, researchers started exploring the feasibility of using GAs for pavement layer moduli backcalculation [14-18, 32, 33]. First, the fundamentals of GA theory are briefly discussed.

In the Simple Genetic Algorithms (SGA) evolutionary search process (see the 'GA module' in Fig. 4), the population size is selected and all the individuals in the population are randomly initialized. During each generation, the solution represented by each individual is evaluated, and solutions are selected for reproduction based on their fitness. Selection embodies the principle of 'survival of the fittest', wherein good solutions are selected for reproduction while bad solutions are eliminated. The fitness value determines the 'goodness' of a solution. The selected solutions then undergo recombination under the action of the crossover and mutation operators. The iterative process of evaluation and genetic manipulation is continued until convergence is reached [30].

In his original work, Holland [31] established the basic theoretical foundation of the genetic algorithms by the use of the schema theory. The Schema Theorem first divides the search space into subspaces and then quantifies the subspaces and explains the movement mechanism of individuals between subspaces.

The overall framework for NGOT is constructed in MATLAB ${ }^{\circledR}$ and the GA module in NGOT is based on the Genetic Algorithm Optimization Toolbox (GAOT) developed at North Carolina State University and implemented in MATLAB ${ }^{\circledR}$ [34].

Backcalculation of pavement layer moduli from FWD deflections can be treated as a global optimization problem where the objective is to determine the unknown pavement layer moduli that minimize the difference between measured and computed deflections. In this paper, the implementation of NGOT is discussed for a three-layered flexible pavement structure (see Fig. 1) although it can be used for other pavement types with varying number of layers owing to its flexible and integrated modular systems approach. Although 
the deflection-based objective function can be defined in a number of ways, a simple objective function representing sum of the squared differences between measured and computed deflections as shown in Eq. 11 was selected for this study (where $n=6$ ):

$$
f=\sum_{i=1}^{n}\left(D_{i}-d_{i}\right)^{2}
$$

$$
\text { Fitness }=1 /(1+f)
$$

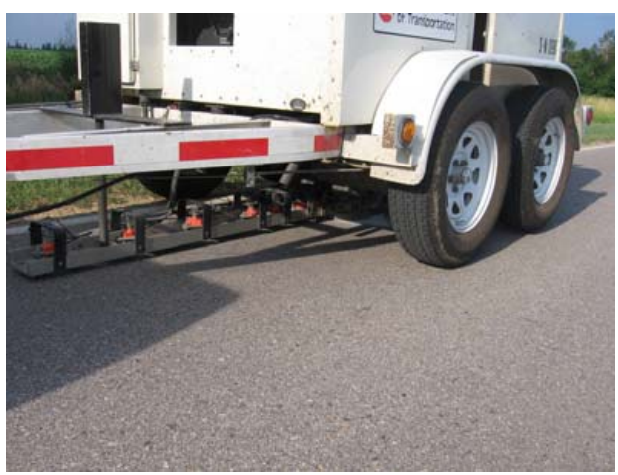

Fig. (1). Trailer-mounted Falling Weight Deflectometer (FWD) equipment.

The input variables to NGOT (see Fig. 4) include six FWD measured deflections (see Fig. 2), AC surface and base layer thicknesses and the corresponding ranges of pavement layer moduli. The use of ranges of values for the optimized unknowns rather than seed values used in the conventional backcalculation approach makes the search for an optimal solution more powerful in the GA approach since a global solution can be obtained and divergence or local optima can be prohibited when compared to conventional methods.

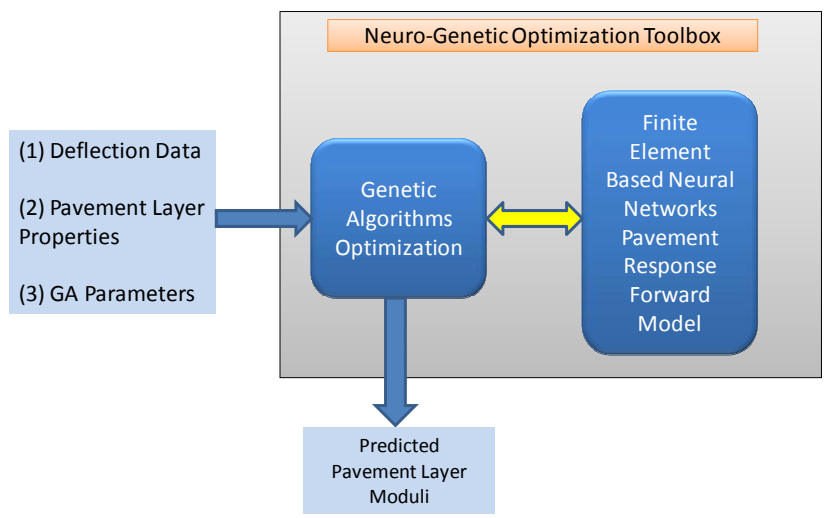

Fig. (2). Neuro-genetic hybrid optimization framework.

The GA module implemented in this study is capable of using either a floating point representation or a binary representation. First, the starting population is randomly generated. Each individual in the population, representing a set of pavement layer moduli, is passed on to the ANN module (which is described in the next section) for computing deflections which are then passed back to the GA module for fitness evaluation. Using the fitness function (Eq. 12), the GA module performs simulated evolution to determine the fitness of the solution strings.

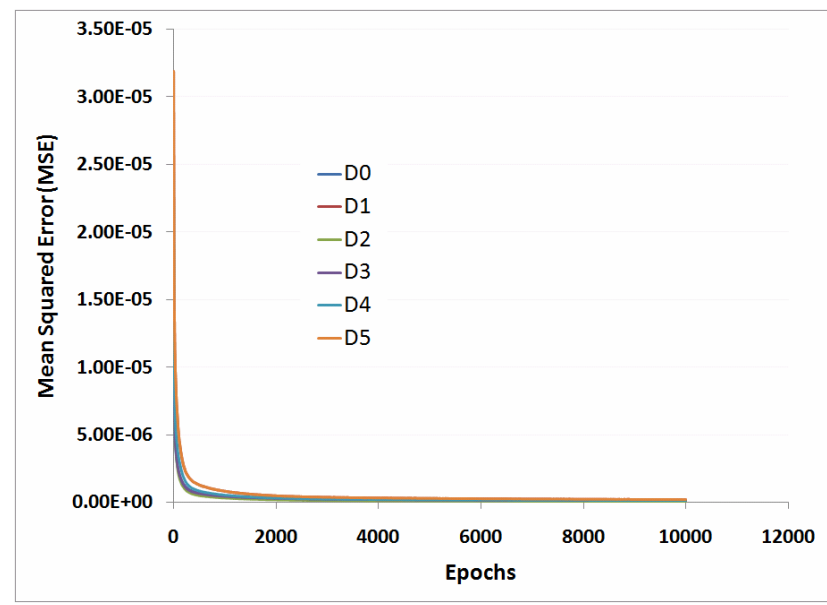

Fig. (3). NN surrogate forward model training progress.

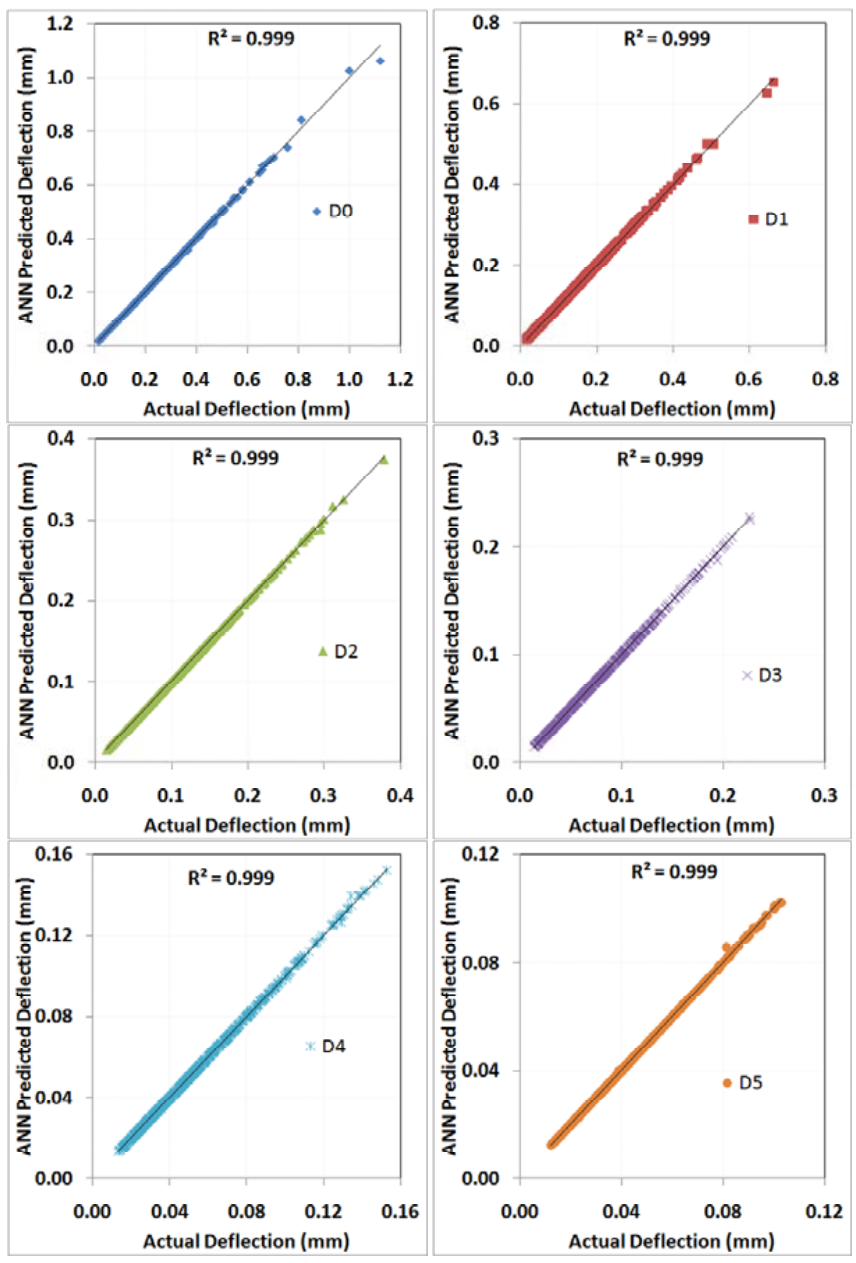

Fig. (4). ANN surrogate forward model prediction accuracy.

In the current GA implementation, it is possible to use a variety of crossover and mutation functions which include arithmetic crossover, heuristic crossover, simple crossover for crossover operator and boundary mutation, multi-nonuniform mutation, non-uniform mutation, and uniform mutation for mutation operator. Similarly, the implemented selection schemes include: roulette wheel, normalized geometric select, and tournament [34]. 
References [17, 18], and [35] discuss the selection of optimal GA parameters for the pavement moduli backcalculation problem. These studies provided some guidelines in optimizing the GA parameters and operators for the current study. Apart from that, a preliminary sensitivity study was conducted to determine the optimal settings for the GA module. The current study described in this paper was conducted using the normalized geometric selection scheme with a probability of 0.08 , arithmetic crossover and nonuniform mutation operators with variable probabilities. The size of the population and generation size were set to 80 and 100, respectively. Since, the ANN and FE modules are interlinked, they are presented together in the next section.

\section{Finite Element Modeling and Neural Networks}

The NN module is an important component of the NGOT which significantly reduces the computational time required for forward calculation of deflections for each of the individuals in the generation. A brief discussion on the ANN background is first presented followed by details related to the development of $\mathrm{NN}$ based surrogate forward models incorporated into NGOT.

NNs are parallel connectionist structures constructed to simulate the working network of neurons in human brain. They attempt to achieve superior performance via dense interconnection of non-linear computational elements operating in parallel and arranged in a pattern reminiscent of a biological neural network. The perceptrons or processing elements and interconnections are the two primary elements which make up a neural network. A single perceptron is mathematically represented as follows [36]:

$$
y_{k}=\varphi\left(v_{j}\right)=\varphi\left(\sum_{i=1}^{n} x_{i} w_{i j}-b_{j}\right)
$$

where $x_{i}$ is input signal, $w_{i j}$ is synaptic weight, $b_{j}$ is bias value, $v_{j}$ is activation potential, $\varphi()$ is activation function, $y_{k}$ output signal, $n$ is the number of neurons for previous layer, and $k$ is the index of processing neuron.

Multilayer perceptrons (MLPs), frequently referred to as multi-layer feedforward neural networks, consist of an input layer, one or more hidden layer, and an output layer. Learning in a MLP is an unconstrained optimization problem, which is subject to the minimization of a global error function depending on the synaptic weights of the network [21]. For a given training data consisting of input-output vectors, values of synaptic weights in a MLP are iteratively updated by a learning algorithm to approximate the target behavior. This update process is usually performed by backpropagating the error signal layer by layer and adapting synaptic weights with respect to the magnitude of error signal [21]. Reference [37] presented the first backpropagation (BP) learning algorithm for use with MLP structures. A general schematic of a multi-layer feedforward neural networks with one output neuron trained using error BP learning algorithm is displayed in Fig. (5).

The backpropagation training algorithm for a simple three-layer MLP structure (one input layer, one hidden layer, and one output layer) is described as follows. The network is initially presented with an input vector $\left(x_{1}, x_{2}, x_{3}, \ldots x_{N}\right)$ augmented by a bias $x_{0}=1$. The net activations of the hidden neurons and the outputs from the hidden layer are calculated as follows:

$$
I_{j}=\varphi\left(\text { neth }_{j}\right)=\varphi\left(\sum_{i=0}^{N} v_{j i} x_{i}\right)
$$

where $i$ varies from 0 to $\mathrm{N}$ and $j$ varies from 1 to $\mathrm{L}$ hidden neurons. The synaptic weights of the interconnections between the inputs and the hidden neurons are represented by $v_{j i}$. Among the nonlinear activation functions, the sigmoid (logistic) function is the most usually employed in ANN application. The presence of a nonlinear activation function, $\varphi()$, is important because, otherwise, the input-output relation of the network could be reduced to that of a single-layer perceptron. The computation of the local gradient for each neuron of the multilayer perceptron requires that the function $\varphi()$ be continuous. In other words, differentiability is the only requirement that an activation function would have to satisfy. The sigmoid function is a bound, monotonic, nondecreasing function that provides graded, nonlinear response within a specified range, 0 to 1 . The sigmoid nonlinear activation function is given by:

$$
\varphi\left(\text { neth }_{j}\right)=\frac{1}{1+\exp \left(-\beta \text { neth }_{j}\right)}
$$

where $\beta$ is a parameter defining the slope of the function. The net activations for the neurons in the output layer and the outputs are calculated as follows:

$$
y_{k}=\varphi\left(\text { neto }_{k}\right)=\varphi\left(\sum_{j=0}^{L} w_{k j} I_{j}\right)
$$

where $\mathrm{k}$ varies from 1 to $\mathrm{M}$ output neurons. The synaptic weights of the interconnections between the hidden neurons and the output neurons are represented by $w_{k j}$. The system error is then computed by comparing the actual outputs $\left(y_{k}\right)$ with the desired outputs $\left(d_{k}\right)$. The error terms for the output neurons $\left(\boldsymbol{\delta}_{k}^{o}\right)$ and the hidden neurons $\left({ }^{h}\right)$ are given by:

$\delta_{k}^{o}=\left(d_{k}-y_{k}\right) \varphi^{\prime}\left(\right.$ neto $\left._{k}\right)$

$\delta_{j}^{h}=\varphi^{\prime}\left(\right.$ neth $\left._{j}\right) \sum_{k=1}^{M} \delta_{k}^{o} w_{k j}$

where the sigmoid activation function is differentiated as follows:

$$
\begin{gathered}
\varphi^{\prime}\left(\text { neto }_{k}\right)=\varphi\left(\text { neto }_{k}\right)\left(1-\varphi\left(\text { neto }_{k}\right)\right)=y_{k}\left(1-y_{k}\right) \\
\varphi^{\prime}\left(\text { neth }_{j}\right)=\varphi\left(\text { neth }_{j}\right)\left(1-\varphi\left(\text { neth }_{j}\right)\right)=I_{j}\left(1-I_{j}\right)
\end{gathered}
$$

Then, the synaptic weights are updated for each neuron in the hidden layer and the output layer. The backpropagation algorithm essentially changes synaptic weights along the negative gradient of error energy function; thus, weight changes are proportional to the magnitude of error energy. 
The formulations for weight updates in the output layer and the hidden layer are given as:

$$
\begin{aligned}
& w_{k j}(t+1)=w_{k j}(t)+\eta \delta_{k}^{o} I_{j}+\alpha\left[w_{k j}(t)-w_{k j}(t-1)\right] \\
& v_{j i}(t+1)=v_{j i}(t)+\eta \delta_{j}^{h} x_{i}+\alpha\left[v_{j i}(t)-v_{j i}(t-1)\right]
\end{aligned}
$$

where $\eta$ is the learning rate parameter that can be selected from the range [0,1] and $\alpha$ indicates momentum term varying within $[0,1]$.

In the BP learning algorithm, the error energy used for monitoring the progress toward convergence is the generalized value of all errors that is calculated by the leastsquares formulation and represented by a Mean Squared Error (MSE) as follows [36]:

$$
M S E=\frac{1}{M P} \sum_{1}^{P} \sum_{k=1}^{M}\left(d_{k}-y_{k}\right)^{2}
$$

where $\mathrm{M}$ is the number of neurons in the output layer and $\mathrm{P}$ represents the total number of training patterns. Other performance measures such as the Root Mean Squared Error (RMSE), Average Absolute Error (AAE), etc. are also used.

A NN-based forward calculation procedure was developed to map the relation between input layer thicknesses and moduli and output surface deflections for passing on to the GA module during fitness evaluation. The goal is to simulate FWD loading using a numerical model for a wide variety of layer thicknesses and combinations of layer moduli encountered in the field resulting in a comprehensive synthetic solution set. A 2-D axi-symmetric FE program [38] commonly used in the structural analysis of flexible pavements was employed to generate a comprehensive synthetic database of moduli-deflection solutions for wide ranges of layer thicknesses and layer moduli. Numerous research studies have validated that this FE model [38] provides a realistic pavement structural response prediction for both highway and airfield pavements by incorporating stress-sensitive geomaterial models, the typical hardening behavior of nonlinear unbound aggregate bases and softening nature of subgrade soils under increasing stress states, and Mohr-Coulomb failure criteria to limit material strength [39].

The synthetic database of FE solutions constituted the training and testing sets for developing NN-based models for rapid forward analysis of flexible pavements. A generic three-layer flexible pavement structure consisting of Asphalt Concrete (AC) surface layer, unbound aggregate base layer, and subgrade layer was modeled using the FE software [38].

The top surface AC layer was characterized as a linear elastic material with Young's Modulus, $\mathrm{E}_{\mathrm{AC}}$, and Poisson ratio, $v$. The $\mathrm{K}-\theta$ model [40] was used as the non-linear characterization model for the unbound aggregate layer:

$$
E_{R}=K \theta^{n}
$$

where $E_{R}$ is resilient modulus (MPa), $\theta=\sigma_{1}+\sigma_{2}+\sigma_{3}=\sigma_{1}+$ $2 \sigma_{3}=$ bulk stress, and $K$ and $n$ are multiple regression constants obtained from repeated load triaxial test data on granular materials. Based on the work reported in [41], $K$ and $n$ model parameters can be correlated to characterize the non-linear stress dependent behavior with only one model parameter. Thus, good quality granular materials, such as crushed stone, show higher $K$ and lower $n$ values, whereas the opposite applies for lower quality aggregates.

Fine-grained subgrade soils were modeled using the commonly used bi-linear resilient modulus model [4]:

$$
\begin{array}{ll}
E_{R}=E_{R i}+K_{1} \cdot\left(\sigma_{d}-\sigma_{d i}\right) & \text { for } \sigma_{d}<\sigma_{d i} \\
E_{R}=E_{R i}+K_{2} \cdot\left(\sigma_{d}-\sigma_{d i}\right) & \text { for } \sigma_{d}>\sigma_{d i}
\end{array}
$$

where $E_{R i}$ is the breakpoint resilient modulus, $\sigma_{d}$ is the breakpoint deviator stress $\left(\sigma_{d}=\sigma_{l}-\sigma_{3}\right), \sigma_{d i}$ is the breakpoint deviator stress, and $K_{1}$ and $K_{2}$ are statistically determined coefficients from laboratory tests. $E_{R i}$ can be used to classify fine-grained subgrade soils as being soft, medium or stiff.

Thus, asphalt concrete modulus, $E_{A C}$, granular base $\mathrm{K}-\theta$ model parameter $K$, and the subgrade break-point resilient moduli, $E_{R i}$, were used as the layer stiffness inputs for all the FE runs. The $40-\mathrm{kN}$ wheel load was applied as a uniform pressure of $552 \mathrm{kPa}$ over a circular area of radius $150 \mathrm{~mm}$ simulating the FWD loading. A comprehensive FE synthetic database was generated by varying the AC layer thickness (in the range of 75 to $700 \mathrm{~mm}$ ), aggregate base layer thickness (in the range of 100 to $550 \mathrm{~mm}$ ), $E_{A C}$ (in the range of 6.9 to $41.5 \mathrm{GPa}$ ), $\mathrm{K}$ (in the range of 21 to $82 \mathrm{MPa}$ ), and $E_{R i}$ (in the range of 7 to $105 \mathrm{MPa}$ ) for $\mathrm{NN}$ training and testing. Independent datasets were used for $\mathrm{NN}$ training and testing.

A range of $(-0.2,+0.2)$ was used for random initialization of all synaptic weight vectors in the network for this study. A smooth learning curve was achieved with a learning-rate parameter $(\eta)$ of 0.001 . Several simulation runs were performed on the network to study its behavior with respect to varying network parameters. More details related to the development of optimal $\mathrm{NN}$ configuration for forward analysis can be found in [13]. In this study, the 5-40-40-6 architecture was chosen as the best architecture for the $\mathrm{NN}$ forward model based on its lowest training and testing MSEs for all six deflection output variables. The five inputs correspond to three layer stifnesses, AC layer thickness and base layer thickness. The NN training progress is shown in Fig. (3) for all six predicted output deflections. Fig. (4) depicts the prediction ability of the 5-40-40-6 network at the 10,000th epoch. Average Absolute Errors (AAEs) were calculated as sum of the individual absolute relative errors divided by the number of independent testing patterns $(1,500$ in this case).

$$
\begin{aligned}
& \text { Average Absolute Error }(A A E), \%= \\
& \sum_{i=1}^{n}\left|\frac{y_{\text {actual }}-y_{\text {predicted }}}{y_{\text {actual }}}\right|_{i} * 100
\end{aligned}
$$

Where $i$ is the $i$ th testing pattern among $n$ testing patterns. The AAE values for NN predicted output deflections were in the range of $0.2 \%-0.4 \%$ with $\mathrm{R}^{2}$ values above 0.999 , indicating proper training and excellent prediction performance of the NN surrogate forward calculation model.

Such NN forward surrogate models integrated into the inversion process offer a number of advantages over the traditional methods, due to their generalization capabilities, 
massive parallelism and potential to offer real-time solutions, thus making them perfect tools for rapidly analyzing the routinely collected FWD deflection data.

In NGOT, the developed ANN models, which have inherited the sophistication and non-linear, stress-sensitive modeling capabilities of the FE module, replace the need to run the FE software for fitness evaluation of each individual in the population, thus resulting in significant savings in terms of time and computational resources.

\section{Performance of Neuro-Genetic Optimization Approach Using Synthetic and Field Data}

The performance of NGOT was first evaluated using hypothetical data covering wide ranges of layer thicknesses and FWD deflections commonly encountered in the field. A total of about 100 datasets were independently selected from the comprehensive synthetic FE solutions database to assess the prediction accuracy of NGOT. The performance of NGOT in backcalculating flexible pavement layer moduli is reported in Fig. (5).
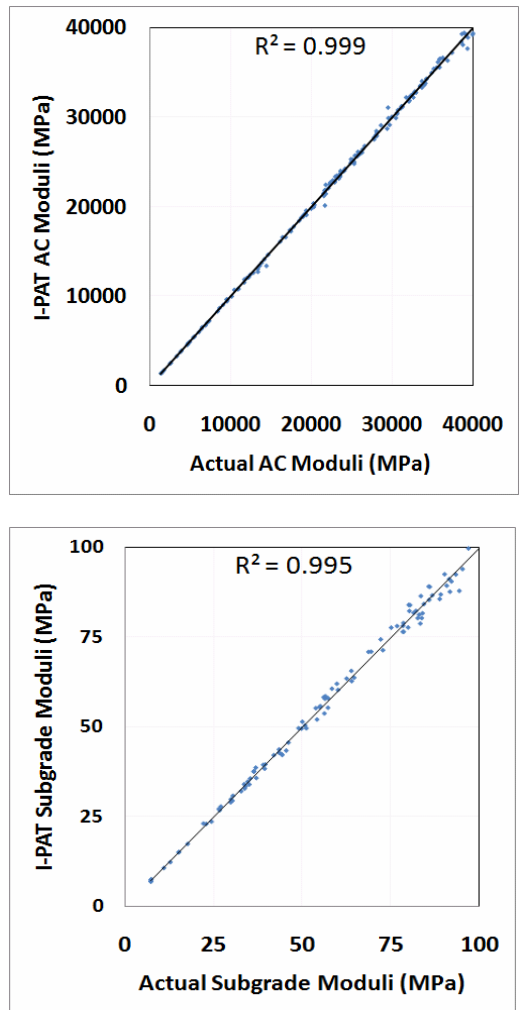

Fig. (5). NGOT prediction accuracy - asphalt concrete moduli and subgrade moduli.

Representative fitness curves depicting the growth of individual fitnesses through generations for typical pavement sections are displayed in Fig. (6). These progress curves confirm that no premature convergence was reached during the analyses. It is seen that the AC and non-linear subgrade moduli values predicted by NGOT compare very well with the actual moduli as demonstrated by very high $R^{2}$ values (above 0.99). These results demonstrate the feasibility of using intelligent evolutionary optimization approach for backcalculating non-linear pavement layer moduli from NDT deflection data.

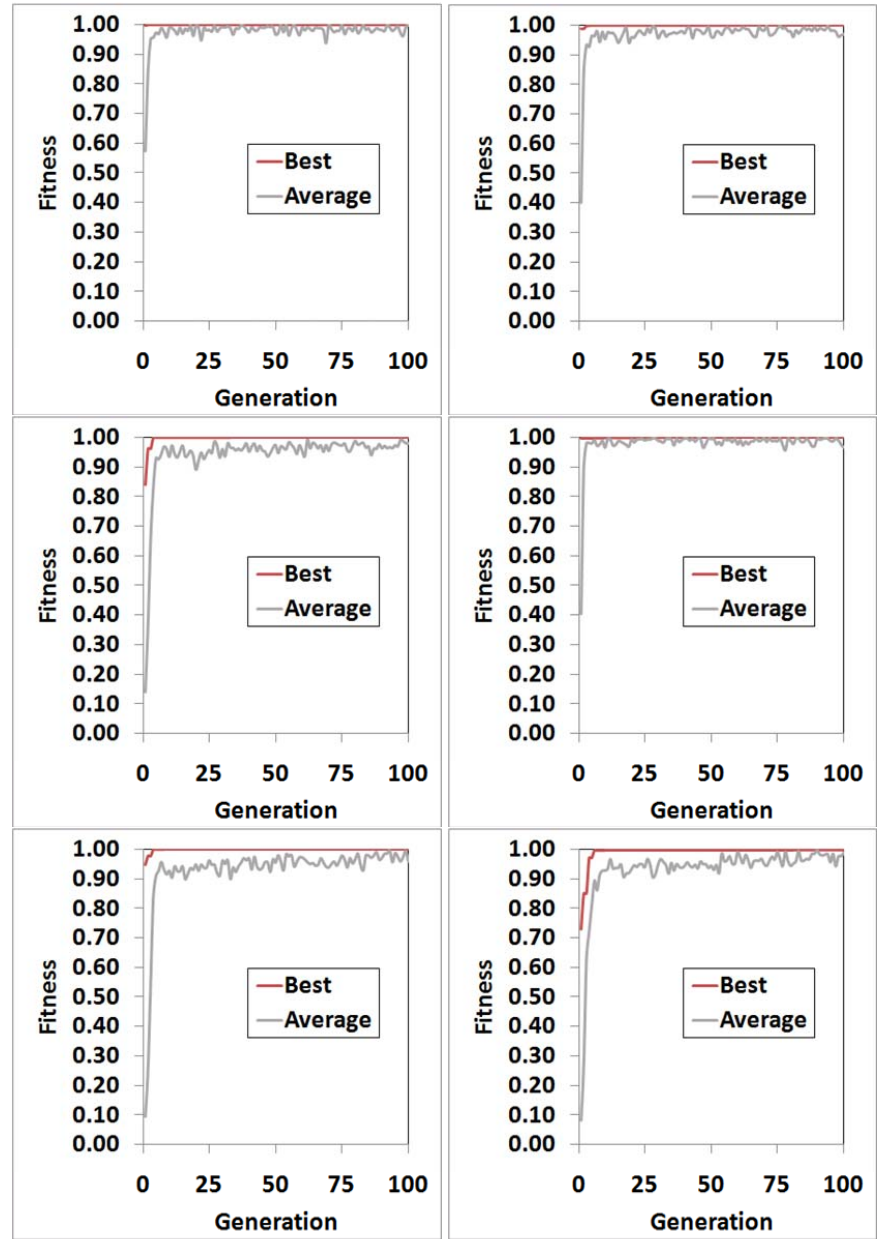

Fig. (6). Neuro-genetic algorithms' representative fitness curves using synthetic test data.

The next step is to assess the performance of developed Neuro-Genetic Optimization Toolbox using actual field data. FWD data collected from a typical flexible pavement in Henry County, Illinois [12] was used. The thicknesses of AC surface layer and granular base layer were $90 \mathrm{~mm}$ (3.5 in.) and $560 \mathrm{~mm}$ (22 in.), respectively for the analyzed pavement section. A total of 79 FWD drops at $40 \mathrm{kN}$ (9 kip) force amplitude was carried out along the length of the in-service county road. FWD deflection data for only the first four radial offsets (D0, D300, D600, and D900) were available. The recorded maximum FWD deflections (D0) along the pavement section are plotted in Fig. (7). The data shows considerable variability with D0 values ranging from 0.51 $\mathrm{mm}$ to $1.32 \mathrm{~mm}$ and is therefore considered a good case for examining the robustness of NGOT.

The Area Under Pavement Profile (AUPP) (see Fig. 8) is a Deflection Basin Parameter (DBP) that has been successfully used as a measure of pavement stiffness (lower AUPP corresponds to higher stiffness and vice versa) as well as to relate to horizontal strain at the bottom of the AC layer $\left(\varepsilon_{\mathrm{AC}}\right)$. Based on FE synthetic database [42], the $\varepsilon_{\mathrm{AC}}$ has been 
correlated with the AUPP term for conventional and full-depth flexible pavements. References [43] and [44] validated the $\varepsilon_{\mathrm{AC}}$ - AUPP relations based on the analyses of $\mathrm{Mn} / \mathrm{ROAD}$ field data (FWD testing and AC strain gauge readings). They were found to be valid at various load levels. The AUPP values (converted from inches to $\mathrm{mm}$ ) at the corresponding FWD drop locations are plotted on Fig. (9).

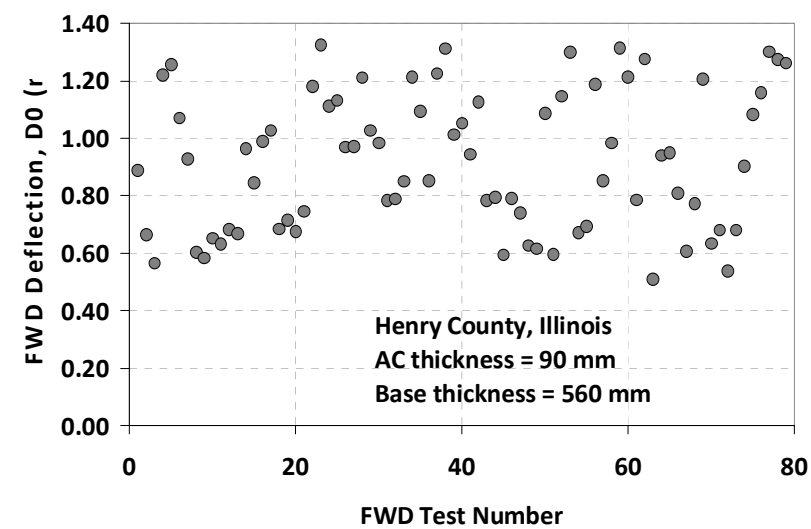

Fig. (7). Variability in maximum FWD surface deflection in Henry County FWD data.

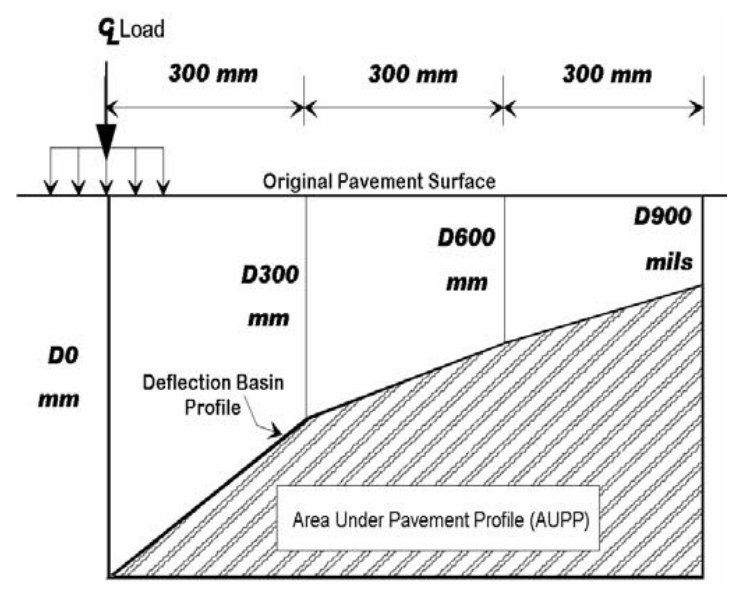

Fig. (8). Area Under Pavement Profile (AUPP).

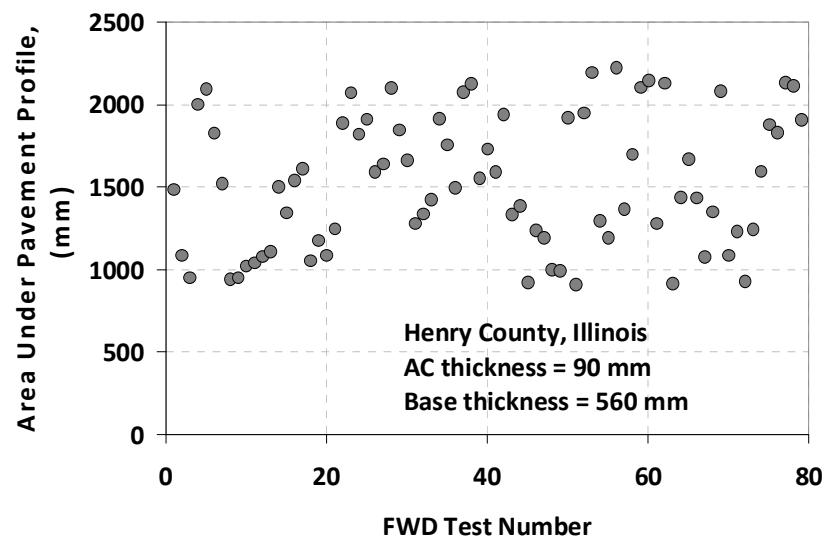

Fig. (9). Variability in AUPP deflection basin parameter in Henry County FWD data.

In a previous University of Illinois study, Reference [45] developed direct plot procedures and algorithms for backcal- culating pavement layer and subgrade moduli from $40-\mathrm{kN}$ (9-kip) FWD test results using comprehensive FE synthetic database. The AC thicknesses ranged from 25 to $200 \mathrm{~mm}$ (1 to 8 in.) and the granular base thicknesses ranged from 100 to $600 \mathrm{~mm}$ (4 to $24 \mathrm{in}$.). For a conventional AC pavement, the regression algorithms are [45]:

$$
\begin{aligned}
& E_{R i}=24.1-5.08 * D_{36}+0.28 * D_{36}{ }^{2} \\
& R^{2}=0.97 \quad S E E=0.76 \\
& L O G E_{A C}=1.48+1.76 * L O G\left(\frac{A R E A}{D_{0}}\right)+0.26 *\left(\frac{A R E A}{T_{A C}}\right) \\
& R^{2}=0.95 \quad S E E=0.110 \\
& \text { Where } \\
& \mathrm{E}_{\mathrm{AC}} \quad=\mathrm{AC} \text { modulus }(\mathrm{ksi}) \\
& \mathrm{E}_{\mathrm{Ri}}=\text { Subgrade soil resilient modulus at a } \\
& \text { repeated deviator stress of } 6.2 \mathrm{psi}(\mathrm{ksi}) \\
& \mathrm{D}_{0} \quad=\text { surface deflection @ } 0 \text { inches from center } \\
& \text { of loading plate (mils) } \\
& \mathrm{D}_{12}=\text { surface deflection @ } 12 \text { inches from center } \\
& \text { of loading plate (mils) } \\
& \mathrm{D}_{24} \quad=\text { surface deflection @ } 24 \text { inches from center } \\
& \text { of loading plate (mils) } \\
& \mathrm{D}_{36}=\text { surface deflection @ } 36 \text { inches from center } \\
& \text { of loading plate (mils) } \\
& \mathrm{T}_{\mathrm{AC}}=\text { thickness of the } \mathrm{AC} \text { layer (inches) } \\
& \text { AREA }=\text { a deflection basin parameter (inches) } \\
& {\left[\text { AREA (in.) }=6\left(1+2\left(\mathrm{D}_{12} / \mathrm{D}_{0}\right)+2\left(\mathrm{D}_{24} / \mathrm{D}_{0}\right)+\left(\mathrm{D}_{36} / \mathrm{D}_{0}\right)\right)\right]}
\end{aligned}
$$

The NGOT pavement layer moduli predictions for the Henry County FWD data are plotted against the statistical model predictions in Fig. (10). It is seen that NGOT predictions are consistent with the statistical model predictions for the analyzed field data which show considerable variability.

The developed NGOT backcalculation tool was also validated using actual field data acquired from an airport flexible pavement test section referred to as MFC at the U.S. National Airport Pavement Test Facility (NAPTF). MFC is a conventional granular base flexible pavement resting over a medium-strength subgrade. It consists of 127-mm (5-in.) thick HMA surface course, 200-mm thick crushed stone granular base, 307-mm thick granular subbase on top of the subgrade. A CL-CH soil classification (ASTM Unified Soil Classification System) material known as Dupont Clay (DPC) was used for the medium-strength subgrade (target California Bearing Ratio of 8). The naturallyoccurring sandy-soil material (SW-SM soil classification) at the full-scale test site underlies the subgrade layer. Detailed information related to NAPTF flexible test sections, material properties, and analysis of NDT data are found in reference [46]. The MFC NDT data referenced in this paper is accessible for download at the Federal Aviation Administration (FAA) Airport Technology Website: www.airporttech.tc.faa.gov. 


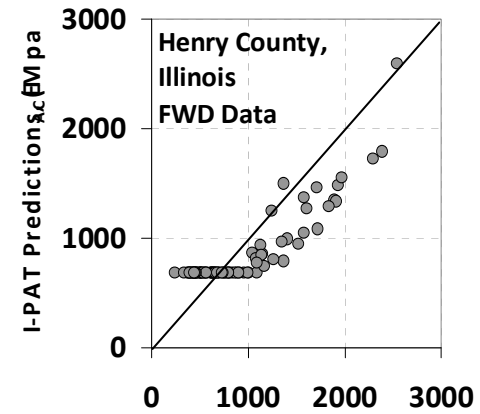

Statistical Algorithm Predictions, $\mathrm{E}_{\mathrm{AC}}$ (Mpa)

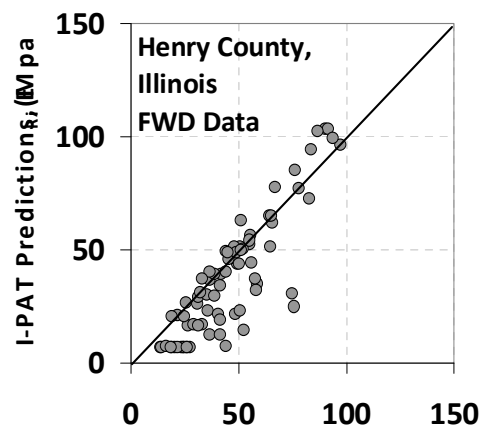

Statistical Algorithm Predictions, $\mathrm{E}_{\mathrm{Ri}}$ (Mpa)

Fig. (10). Comparison of statistical algorithm and neuro-genetic moduli predictions - Henry County FWD data.

The NGOT pavement layer moduli predictions for MFC test section are shown in Fig. (11). The NGOT backcalculation results were then compared with three existing backcalculation methods: (1) FE-based Statistical Algorithms (see Eq. 17) [45], (2) BAKFAA [47], and (3) WESDEF [48]. Both BAKFAA and WESDEF are conventional deflectionbasin matching backcalculation programs based on elastic layered theory.

The NGOT backcalculated flexible pavement layer moduli for MFC are plotted against those predicted by the other three methods in Fig. (12). The neuro-genetic moduli predictions seem to be consistent and agreeable with those predicted by the existing approaches. Note that the ELP-based backcalculation programs, BAKFAA and WESDEF, assume the subgrade to be linear elastic while both the Regression Algorithms and the NGOT prediction methodology consider the non-linear stress-dependent subgrade properties. However, the NGOT methodology is based on a population-based stochastic optimization technique which is different from the approach employed by all the other three methods.

A major advantage of the proposed integrated systems approach is that emerging concepts and methodologies that are continually evolving in the individual modular applications can be easily incorporated within the overall framework to enhance the overall robustness of the toolbox. It was demonstrated that NGOT can successfully predict the flexible pavement surface layer moduli and non-linear subgrade moduli using both hypothetical data as well as actual field data.
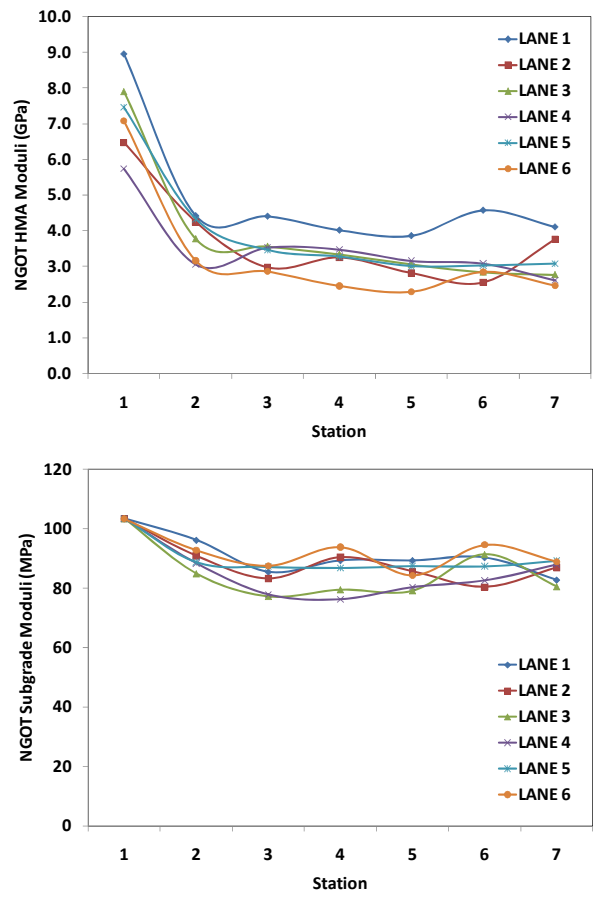

Fig. (11). Neuro-genetic pavement layer moduli predictions for MFC test section.
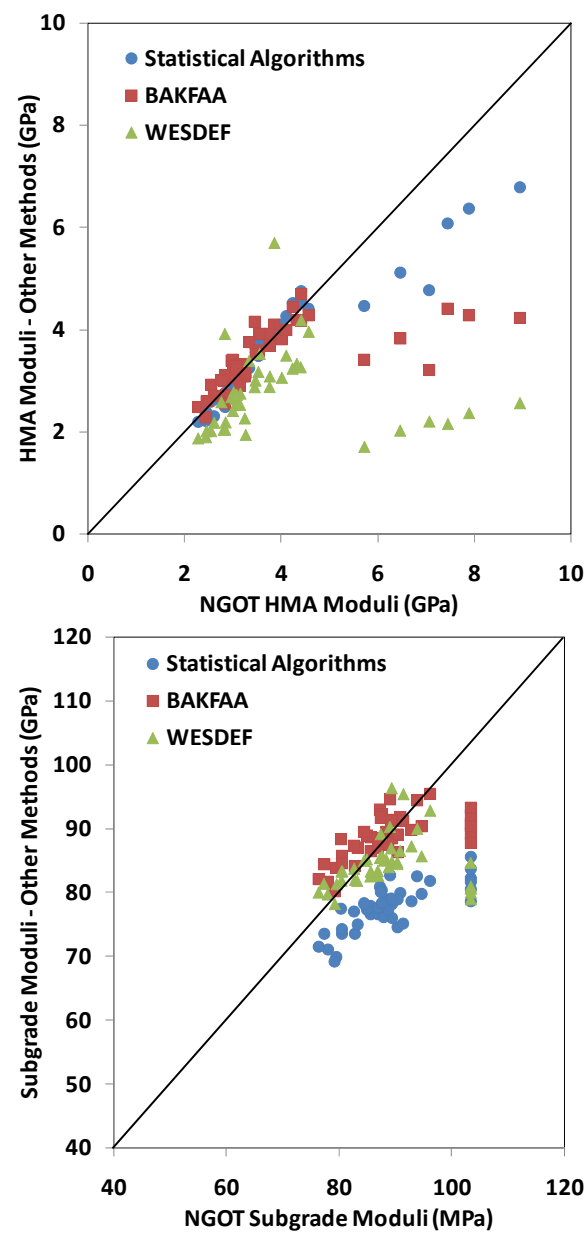

Fig. (12). Comparison of neuro-genetic pavement layer moduli predictions with other methods - MFC FWD data. 


\section{REFERENCES}

[1] 2005 Report Card for America's Infrastructure. Available: http://www.asce.org/reportcard/2005/index.cfm. (URL) [Accessed Jan. 20, 2009].

[2] Office of Highway Policy Information. Highway Statistics 2000, Federal Highway Administration, Washington, DC, 2004.

[3] FAA, Use of Nondestructive Testing in the Evaluation of Airport Pavements, FAA Advisory Circular No. 150/5730-11A, Office of Airport Safety and Standards, Federal Aviation Administration, U.S. Department of Transportation, Washington, DC, 2004.

[4] A. J. Bush III and G. Y. Baladi, Nondestructive testing of pavements and backcalculation of moduli, ASTM Special Technical Publication (STP) 1026, 1989.

[5] S. D. Tayabji and E. O. Lukanen, Nondestructive testing of pavements and backcalculation of moduli, ASTM Special Technical Publication (STP) 1375, 2000.

[6] R. L. Lytton, "Backcalculation of layer moduli, state of the art," In: NDT of pavements and backcalculation of moduli, A. J. Bush and G. Y. Baladi, Eds., Vol. 1, ASTM Special Technical Publication (STP) 1026, 1989, pp. 7-38.

[7] P. Ullidtz and N. F. Coetzee, "Analytical Procedures in Nondestructive Testing Pavement Evaluation," Trans. Res. Rec., vol. 1482, pp. 61-66, 1995

[8] N. Sivaneswaran, S. L. Kramer, and J. P. Mahoney, "Advanced backcalculation using a non-linear least squares optimization technique," Trans. Res. Rec., vol. 1293, pp. 93-102, 1991.

[9] M. S. Hoffman, and M. R. Thompson, "Backcalculating nonlinear resilient moduli from deflection data," Trans. Res. Rec., vol. 852, pp. 42-51, 1982.

[10] J. Uzan, "Advanced backcalculation techniques," In: NDT of pavements and backcalculation of moduli, H. L. Von Quintus, A. J. Bush, and G. Y. Baladi, Eds., Vol. 2, ASTM Special Technical Publication (STP) 1198, 1994, pp. 3-37.

[11] R. W. Meier, and G. J. Rix, "Backcalculation of flexible pavement moduli using artificial neural networks," Trans. Res. Rec., 1448, pp. 75-82, 1994.

[12] H. Ceylan, A. Guclu, M. B. Bayrak, and K. Gopalakrishnan, "Nondestructive evaluation of Iowa pavements-Phase I," CTRE, Iowa State University, Ames, IA, CTRE Project 04-177, Dec. 2007.

[13] K. Gopalakrishnan, and M. R. Thompson, "Backcalculation of airport flexible pavement non-linear moduli using artificial neural networks," in Seventeenth International FLAIRS Conference, Miami Beach, Florida, May, 2004.

[14] T. F. Fwa, C. Y. Tan, and W. T. Chan, "Backcalculation analysis of pavement-layer moduli using genetic algorithms," Trans. Res. Rec., vol. 1570, pp. 134-142, 1997.

[15] M. A. Reddy, K. S. Reddy, and B. B. Pandey, "Backcalculation of pavement moduli using genetic algorithms," J. Highw. Res. Board, vol. 66, pp. 1-10, 2002.

[16] H. M. Park, S. W. Park, and J. J. Hwang, "Use of genetic algorithm and finite element method for backcalculating layer moduli in asphalt pavements," in 86th Annual Meeting of the Transportation Research Board (TRB), Washington, DC, 2007.

[17] W. Alkasawneh, "Backcalculation of pavement moduli using genetic algorithms," Ph.D. dissertation, Department of Civil Engineering, University of Akron, Akron, Ohio, 2007.

[18] O. Peckcan, E. Tutumluer, and M. R. Thompson, "Nondestructive pavement evaluation using ILLI-PAVE based artificial neural network models," Illinois Center for Transportation, Champaign, IL, Research Rep. FHWA-ICT-08-022, Sep. 2008.

[19] K. Gopalakrishnan, and H. Ceylan, "A system of systems approach to transportation infrastructure management," Journal of Information, Intelligence, and Knowledge, 2009, [Epub ahead of print].

[20] S. Terzi, M. Saltan, and T. Yildirim, "Optimization of deflection basin by genetic algorithm and neural network approach," in International Conference on Neural Networks, ICANN/ICONIP, 2003, pp. 662-669.

[21] A. B. Goktepe, E. Agar, and A. H. Lav, "Comparison of Multilayer Perceptron and Adaptive Neuro-Fuzzy System on Backcalculating the Mechanical Properties of Flexible Pavements," ARI: The Bulletin of the Istanbul Technical University, vol. 54, no. 3, pp. 1-13, 2006.

[22] R. W. May, and H. L. Von Quintas, "The quest for a standard guide to NDT backcalculation," in Nondestructive testing of pavements and backcalculation of moduli, vol. 2, ASTM STP 1198, pp. 505$520,1994$.

[23] M. Anderson, "Backcalculation of Composite Pavement Payer Moduli," Ph.D. Dissertation, University of Kentucky, Kentucky, USA, 1988.

[24] S. F. Brown, and J. W. Pappin, "Analysis of pavements with granular bases," Trans. Res. Rec., vol. 810, pp. 17-23, 1981.

[25] M. R. Thompson, and R. P. Elliot, "ILLI-PAVE based response algorithms for design of conventional flexible pavements," Trans. Res. Rec., vol. 1043, pp.50-57, 1985.

[26] N. E. Garg, E. Tutumluer, and M. R. Thompson, "Structural modelling concepts for the design of airport pavements for heavy aircraft," in Fifth International Conference on the Bearing Capacity of Roads and Airfields, Trondheim, Norway, 1998.

[27] Y. J. Chou and R. L. Lytton, "Accuracy and consistency of backcalculated pavement layer moduli," Trans. Res. Rec., vol. 1293, pp. 72-85, 1991

[28] L. Davis (Ed.), Genetic Algorithms and Simulated Annealing, London: Pitman, 1987.

[29] D. E. Goldberg, Genetic Algorithms in Search, Optimization and Machine Learning. Reading, MA: Addison Wesley, 1989.

[30] M. Srinivas and L. M. Patnaik, "Adaptive probabilities of crossover and mutation in genetic algorithms," IEEE Trans. Systems, Man, and Cybernetics, vol. 24, no. 4, pp. 656-667, 1994.

[31] J. H. Holland, Adaption in Natural and Artificial Systems, Ann Arbor: Univ. Michigan Press, 1975.

[32] S. Kameyama, K. Himeno, A. Kasahara, and T. Maruyama "Backcalculation of pavement layer moduli using genetic algorithms," in Eighth International Conference on Asphalt Pavements, University of Washington, Seattle, Washington, 1998.

[33] B. Tsai, V. Kannekanti, and J. T. Harvey, "Application of genetic algorithm in asphalt pavement design," Trans. Res. Rec., vol. 1891, pp. 112-120, 2004

[34] C. Houck, J. Joines, and M. Kay, "A genetic algorithm for function optimization: A Matlab implementation," North Carolina State University, Raleigh, North Carolina, Research Rep. NCSU-IE TR 95-09, 1995.

[35] M. A. Reddy, K. S. Reddy, and B. B. Pandey, "Selection of genetic algorithm parameters for backcalculation of pavement moduli," Int. J. Pavement Eng., vol. 5, no. 2, pp. 81-90, 2004.

[36] S. Haykin, Neural networks: A comprehensive foundation, New Jersey: Prentice-Hall Inc., 1999.

[37] D. E. Rumelhart, G. E. Hinton, and R. J. Williams, "Learning internal representation by error propogation," in: Rumelhart, D.E. Ed, Parallel Distributed Processing, Cambridge, MA, MIT Press, 1986, pp. 318-362.

[38] L. Raad and J. L. Figueroa, "Load response of transportation support systems," Transport. Eng. J. ASCE, vol. 106, no. TE1, pp. 111-128, 1980.

[39] F. Gomez-Ramirez, M. R. Thompson, and M. Bejarano, "ILLIPAVE based flexible pavement design concepts for multiple wheel heavy gear load aircraft," in Ninth International Conference on Asphalt Pavements, Copenhagen, Denmark, 2002.

[40] R. G. Hicks, and C. L. Monismith, "Factors Influencing the Resilient Properties of Granular Materials," Trans. Res. Rec., vol. 345, pp. 15-31, 1971.

[41] G. Rada and M. W. Witczak, "Comprehensive evaluation of laboratory resilient moduli results for granular material," Trans. Res. Rec., No. 810, pp. 23-33, 1981.

[42] H. J. Hill and M. R. Thompson, "Early Life Study of the FA409 Full-Depth Asphalt Concrete Pavement Sections," Unpublished, University of Illinois Research Report, University of Illinois at Urbana-Champaign, Urbana, IL, 1988.

[43] N. Garg and M. R. Thompson, "Mechanistic Empirical Evaluation of The Mn/Road Low Volume Road Test Sections." Transportation Engineering Series No. 96, Civil Engineering Studies, Department of Civil Engineering, University of Illinois at Urbana-Champaign, Urbana, IL, 1998.

[44] C. Alvarez and M. R. Thompson, "Mechanistic Empirical Evaluation of The Mn/Road Mainline Flexible Pavement Sections," Transportation Engineering Series No. 97, Civil Engineering Studies, Department of Civil Engineering, University of Illinois at Urbana-Champaign, Urbana, IL, 1998.

[45] M. R. Thompson, "ILLI-PAVE Based Full-Depth Asphalt Concrete Pavement Design Procedure," in Sixth International Conf. on 
Structural Design of Asphalt Pavements, Ann Arbor, Michigan, 1987.

[46] K. Gopalakrishnan, "Performance analysis of airport flexible pavement subjected to new generation aircraft", Ph.D. dissertation, University of Illinois at Urbana-Champaign, Urbana, IL, USA, December 2004.

[47] G. H. Hayhoe, "LEAF - A New Layered Elastic Computational Program for FAA Pavement Design and Evaluation Procedures," in
2002 Federal Aviation Administration Airport Technology Transfer Conference, Chicago, 2002.

[48] F. J. Van Cauwelaert, D. R. Alexander, T. D. White and W. R. Barker, "Multilayer elastic program for elastic program for backcalculating layer moduli in pavement evaluation," in Nondestructive Testing of Pavements and Backcalculation of Moduli, ASTM STP 1026, 1989.

(C) Kasthurirangan Gopalakrishnan; Licensee Bentham Open.

This is an open access article licensed under the terms of the Creative Commons Attribution Non-Commercial License (http://creativecommons.org/licenses/by-nc/3.0/) which permits unrestricted, non-commercial use, distribution and reproduction in any medium, provided the work is properly cited. 